\section{Chromosomal instability confers poor prognosis in patients with colorectal cancer}

Despite advances in treatment, colorectal cancer remains a major cause of mortality in the developed world; therefore, it is important to identify patients who will benefit most from treatment. Studies have shown that microsatellite instability is of prognostic importance for patients with colorectal cancer. Recent data, however, have suggested that microsatellite instability could be secondary to the effects of chromosomal instability. Walther et al. conducted a systematic review of all published studies on chromosomal instability in patients with colorectal cancer to investigate the prognostic significance of this abnormality.

The authors used PubMed and EmBase to identify 63 studies that stratified survival by chromosomal instability status in patients with colorectal cancer. The main outcome measure was hazard ratio (HR) for death. Of the 10,126 patients included in this analysis, $60 \%$ had chromosomal instability. Chromosomal instability was significantly associated with an increased risk of death in patients with colorectal cancer of all stages (HR 1.45, 95\% Cl 1.35$1.55 ; P<0.001)$ and in patients with stage IIIII colorectal cancer who were candidates for adjuvant chemotherapy (HR 1.45, 95\% Cl 1.27$1.65 ; P<0.001)$. Progression-free survival was similarly affected by chromosomal instability status (HR 1.71, 95\% Cl 1.51-1.94; $P<0.001$ ).

Walther and colleagues conclude that chromosomal instability is associated with a worse prognosis in patients with colorectal cancer and could be of value as a prognostic marker, along with microsatellite instability. Clinical trials are needed to investigate the contribution of each type of genomic instability to prognosis.

Original article Walther A et al. (2008) Association between chromosomal instability and prognosis in colorectal cancer: a meta-analysis. Gut 57: 941-950

\section{Salvage radiotherapy improves survival in men with recurrence after radical prostatectomy}

Although radical prostatectomy can control prostate cancer in the majority of newly diagnosed cases, many individuals experience biochemical recurrence within 5 years and require salvage radiotherapy. The association between salvage radiotherapy and prostatecancer-specific survival has not been evaluated to date. Trock and co-workers have assessed prostate cancer-specific survival following salvage radiotherapy in men who experienced biochemical recurrence.

Data from a cohort of 635 men who underwent prostatectomy during the period 19822004 and experienced biochemical recurrence were retrospectively analyzed. Of these men, 397 received no salvage treatment, 160 received salvage radiotherapy, and 78 received salvage radiotherapy combined with hormonal therapy.

At a median follow-up of 6 years from the time of recurrence, a total of 116 men had died from prostate cancer. Salvage radiotherapy alone or combined with hormonal therapy was associated with significantly longer prostatecancer-specific survival and overall survival than no salvage treatment. Improved survival was confined to men with a prostatespecific antigen (PSA) doubling time of less than 6 months and remained significant after adjustment for Gleason score, postoperative stage, and time to initial PSA recurrence.

The authors conclude that early salvage radiotherapy is associated with improved survival in men with biochemical disease recurrence after radical prostatectomy. These results need to be validated in other settings, however, particularly since results from a recently initiated European clinical trial comparing salvage radiotherapy with adjuvant radiotherapy will not be available for many years.

Original article Trock BJ et al. (2008) Prostate cancer specific survival following salvage radiotherapy vs observation in men with biochemical recurrence after radical prostatectomy. JAMA 299: 2760-2769

\section{Nonmyeloablative stem-cell transplantation can induce long- term remission in relapsed FL}

For patients with follicular lymphoma (FL), nonmyeloablative conditioning followed by allogeneic stem-cell transplantation is an effective therapeutic option; however, its longterm toxicity and efficacy are unknown. Khouri and colleagues have assessed the effects of rituximab-based nonmyeloablative conditioning in a phase II trial. 